Article

\title{
Model Predictive Control for a Recover Water Process
}

\author{
Paolo Mercorelli iD \\ Institute of Product and Process Innovation; Volgershall 1, D-21339 Lueneburg; Leuphana University of \\ Lueneburg; mercorelli@uni.leuphana.de \\ * Correspondence: mercorelli@uni.leuphana.de; Tel.:+49-(0)4131-677-5571
}

\begin{abstract}
The goal of this contribution is an application of the Linear General Model Predictive Control (LGMPC). In this paper, stability of the LGMPC is proven by means of a demonstration of a Theorem stating a sufficient and constructive $\mathrm{c}$ ondition. This condition can be a pplied for calculating the weight matrices of the cost function in the optimisation problem in LGMPC. Lower bounds conditions are found for one of these matrices and then a system with saturation is taken into consideration. The conditions could be interpreted and discussions through physical aspects. The obtained results were tested by means of computer simulations and an example with a recover water process is considered.
\end{abstract}

Keywords: Linear Model Predictive Control; Process Control; Stability

\section{Introduction}

Model predictive control approach is used for improving the tracking of a desired trajectory. Utilizing the linear prediction algorithm for improving the tracking performances of an adaptive controller can be considered a good idea. Using a prediction structure an improvement of the dynamic performances is awaited., so MPC is used in drives control applications [1,2]. MPC is an optimal control approach being able to deal effectively with constraints and multi variable processes in industries. For its being advantageable MPC has been widely used in automotive and process control communities [3].Solving optimization problem on-line however limits the MPC applications by slow dynamic systems [3].Application of MPC to mechatronic systems for servo design attracts attention of many scientists because of the continuous development of microprocessor technology. Mechatronic systems such as electrical motor control [4], two stage actuation system control, machine tool chattering control [5] have shown promising results. There is also a fast development of various advanced techniques integrated with MPC for performance improvement [6]. The used sampling frequency considered in the research literature may be still too slow for general mechatronic systems. Moreover, simulations show, that the existence of modelling error makes the steady-state error being obvious. These both points, in particular, during real-time implementation may influence the performance of mechatronic systems. Calculation of the solution of the MPC in an off-line in an explicit way can be seen, for instance [7], [8]. MPC has been also applied for piezoelectric actuators, see [9]. Finding conditions on the stability is one of the interesting issues in optimisation. The goal of this paper is to find lower bounds of a matrix characterising the cost function to be able to guarantee the stability of the optimal solution. Results, obtained by means of PI controller in [10]are extended in this contribution. Results obtained using optimal algorithms in [11] are organised in the following way. The Section 2 is dedicated to a system recovering water. This contribution shows only the "difference case" for the MPC, although a similar Theorem was proven in [12]. In Section 4 a property of the LGMPC is proved in case without and with saturation. In Section 6 the system shown in Section 2 is taken as a simulated example and the proposed control technique is applied. Conclusions close the paper. 


\section{Mathematical model of the system}

\begin{tabular}{|l|}
\hline The main nomenclature \\
$m_{\text {in }}(t):$ input mass flow $(\mathrm{kg} / \mathrm{sec})$ \\
$m_{o}(t):$ output mass flow $(\mathrm{kg} / \mathrm{sec})$ \\
$m(t):$ mass $(\mathrm{Kg})$ \\
$\frac{d m(t)}{d t}:$ mass flow $(\mathrm{kg} / \mathrm{sec})$ \\
$p(t):$ pressure inside the evaporator $(\mathrm{Pa})$ \\
$T:$ Temperature $(\mathrm{K})$ \\
$V:$ Volume $(\mathrm{l})$ \\
$p_{i}:$ initial pressure $(\mathrm{Pa})$ \\
$p_{d}:$ desired pressure $(\mathrm{Pa})$ \\
$R_{g}:$ vapor constant \\
$A_{w}:$ anti-windup signal \\
$K_{b}:$ weight factor for the anti-windup action \\
$V_{\mathcal{L}}:$ a Lyapunov function \\
$\mathbf{A}_{k}:$ discrete system matrix \\
$\mathbf{B}_{k}:$ discrete input matrix \\
$\mathbf{H}_{k}:$ output matrix \\
$\mathbf{G}_{p}:$ Model predictive control state matrix \\
$\mathbf{F}_{1 p}:$ Model predictive control "dealta" input matrix \\
$\mathbf{F}_{2 p}:$ Model predictive control input matrix \\
\end{tabular}

In Fig. 1 a schematic representation of the considered system is shown. The system basically consists of the following three elements: a boiler, a compressor and an evaporator. In the boiler the waste water remains in the middle and lower part of it and in the upper part a vapor chamber is to be considered in order to host the water vapor which is generated by heating the waste water. At the beginning the mixture (waste water) is heated by a resistor system until the water vapor is present in the vapor chamber. At this time the heating is switched off and the compressor is switched on. The compressor should provide for a high pressure in the evaporator and in the meantime reduce the pressure in the vapor chamber through and a mass flow. This mass flow $\left(m_{0}(t)\right)$ after the condensed phase represents cleaned water in output of the evaporator and it is stilled. More in depth, in the evaporator, the vapor is condensed giving up heat to the waste water. To guarantee that new waste water can enter into the boiler from the upper part of it, the compressor provides a low pressure in the vapor chamber. In this sense, the compressor works as a controller in this process and its output is represented by the mass flow $m_{0}(t)$ with the constraint that $m_{0}(t)>0$. The compressor is equipped with an asynchronous motor which is controlled in frequency using an inverter piloted by a PWM signal which converts the output of the LMPC controller in frequency. In input to LMPC the pressure error is provided. More details of the control scheme will be given in the chapter devoted to the simulation. In any case, the dynamics of the asynchronous motor together with the inverter and all rest of the converters, considered all together, are much faster than the dynamics of the controlled process and in this analysis are not taken into account. Short description of the drain: At the beginning the pressure in the container equals the ambient pressure (circa 1.013 bar). By heating, the pressure increases slowly, as the water begins to evaporate. New water is added by a constant control with floats. The condensed water vapor from the "internal heat exchanger" is now free of foreign matter. For controller design purposes the dynamical model of the system must be considered. As already 


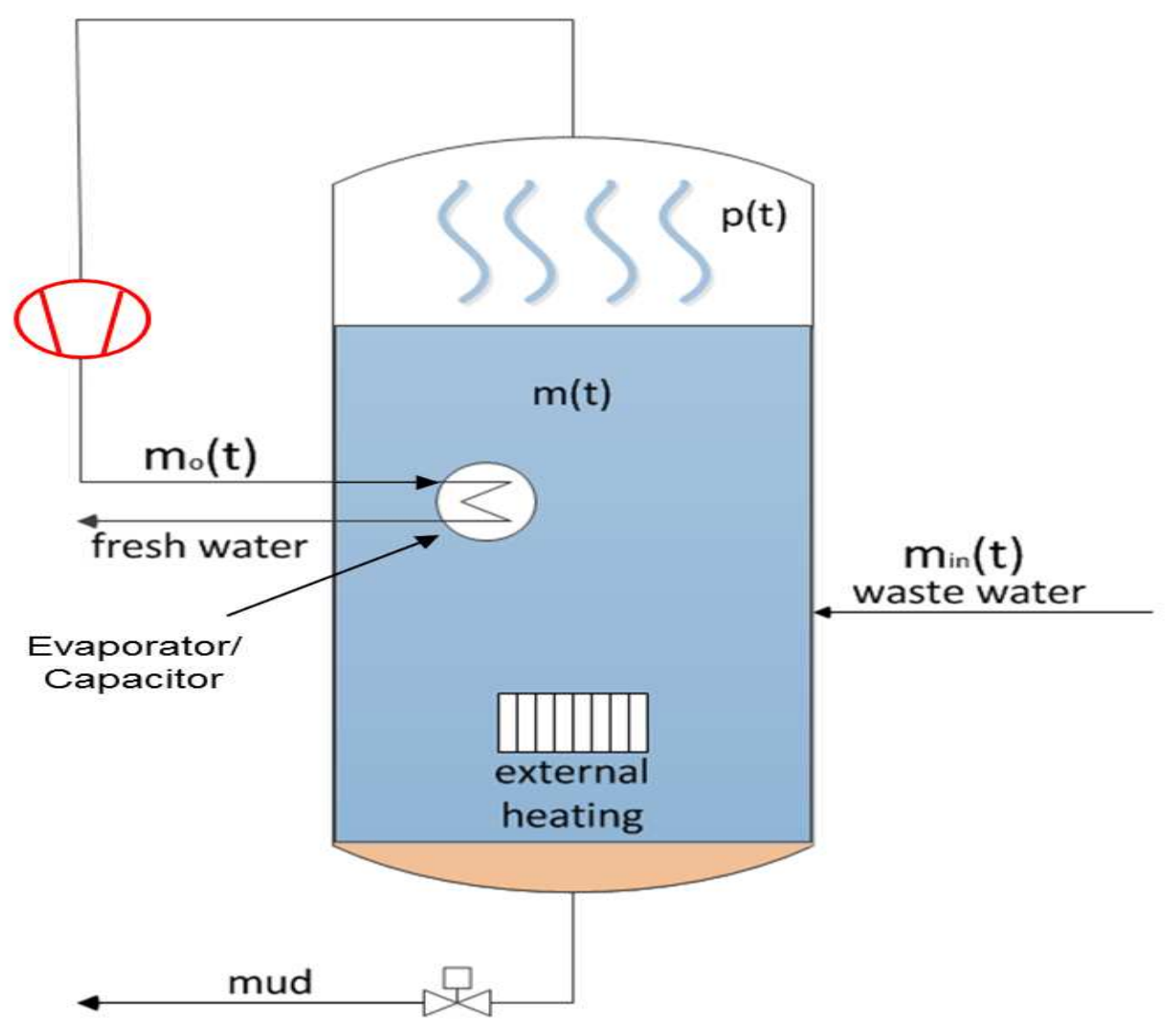

Figure 1. Boiler system

explained, considering that the control process starts after the heating phase and more precisely when the water vapor becomes to be present in the boiler, the following equations can be considered:

$$
\begin{gathered}
\frac{d m(t)}{d t}=m_{i}(t)-m_{o}(t) \\
\frac{d p(t)}{d t}=\frac{d m(t)}{d t} \frac{R_{g} T}{V}
\end{gathered}
$$

38 in which $m_{i}(t)$ is a stepwise positive constant function.

39

Considering the Forward Euler discretisation with sampling time $T_{s}$, the following expression is obtained:

$$
\begin{aligned}
& m(k+1)=T_{s}\left(m_{i}(k)-m_{o}(k)\right)+m(k) \\
& p(k+1)=\left(m_{i}(k)-m_{o}(k)\right) \frac{R_{g} T}{V}+p(k),
\end{aligned}
$$

$40 \quad$ and thus

$$
\underbrace{\left[\begin{array}{c}
m(k+1) \\
p(k+1)
\end{array}\right]}_{\hat{\mathbf{z}}(k+1)}=\underbrace{\left[\begin{array}{cc}
T_{S} & 0 \\
0 & 1
\end{array}\right]}_{\mathbf{A}_{k}} \underbrace{\left[\begin{array}{c}
m(k) \\
p(k)
\end{array}\right]}_{\hat{\mathbf{z}}(k)}+\underbrace{\left[\begin{array}{c}
T_{S} \\
T_{s} R_{g} T \\
V
\end{array}\right]}_{\mathbf{B}_{k}} \underbrace{\left(m_{i}(k)-m_{0}(k)\right)}_{u_{m p c(t)}}
$$




\section{Model predictive control}

In the model approach just two samples are considered:

$$
\hat{\mathbf{z}}(k+1 / k)=\mathbf{H}_{k} \mathbf{A}_{k} \mathbf{z}(k / k)+\mathbf{H}_{k} \mathbf{B}_{k} u_{m p c}(k) .
$$

42

$$
\begin{gathered}
\text { If } \Delta u_{m p c}(k)=u_{m p c}(k)-u_{m p c}(k-1) \text {, then } u_{m p c}(k)=\Delta u_{m p c}(k)+u_{m p c}(k-1) \text { and } \\
\hat{y}_{h}(k+1)=\mathbf{H}_{k} \mathbf{A}_{k} \hat{\mathbf{z}}(k / k)+\mathbf{H}_{k} \mathbf{B}_{k}\left(\Delta u_{m p c}(k)+u_{m p c}(k-1)\right) .
\end{gathered}
$$

where matrix $\mathbf{H}_{k}$ selects the second state variable and thus $y_{h}(t)$ represents the pressure.

$$
\hat{y}(k+2)=\mathbf{H}_{k} \mathbf{A}_{k}^{2} \hat{\mathbf{z}}(k / k)+\mathbf{H}_{k} \mathbf{A}_{k} \mathbf{B}_{k}\left(\Delta u_{m p c}(k)+u_{m p c}(k-1)\right)+\mathbf{H}_{k} \mathbf{B}_{k}\left(\Delta u_{m p c}(k+1)+u_{m p c}(k)\right) .
$$

It is straightforward to show that the following vectorial expression holds:

$$
\hat{\mathbf{Y}}_{h}(k)=\mathbf{G}_{p} \mathbf{x}(k)+\mathbf{F}_{1 p} \Delta \mathbf{U}_{m p c}(k)+\mathbf{F}_{2 p} u_{m p c}(k-1),
$$

where

$$
\hat{\mathbf{Y}}_{h}(k)=\left[\begin{array}{c}
\hat{y}_{h}(k+1) \\
\hat{y}_{h}(k+2)
\end{array}\right], \quad \quad \quad \quad \mathbf{U}_{m p c}(k)=\left[\begin{array}{c}
\Delta u_{m p c}(k) \\
\Delta u_{m p c}(k+1),
\end{array}\right]
$$

and matrices $\mathbf{G}_{p}, \mathbf{F}_{1 p}$ and $\mathbf{F}_{2 p}$ are given by:

$$
\mathbf{F}_{1 p}=\left[\begin{array}{cc}
\mathbf{H}_{k} \mathbf{B}_{k} & \mathbf{0} \\
\mathbf{H}_{k}\left(\mathbf{A}_{k} \mathbf{B}_{k}+\mathbf{B}_{k}\right) & \mathbf{H}_{k} \mathbf{B}_{k}
\end{array}\right], \quad \mathbf{G}_{p}=\left[\begin{array}{c}
\mathbf{H}_{k} \mathbf{A}_{k} \\
\mathbf{H}_{k} \mathbf{A}_{k}^{2}
\end{array}\right], \quad \mathbf{F}_{2 p}=\left[\begin{array}{c}
\mathbf{H}_{k} \mathbf{B}_{k} \\
\mathbf{H}_{k}\left(\mathbf{A}_{k} \mathbf{B}_{k}+\mathbf{B}_{k}\right)
\end{array}\right] .
$$

If the following performance criterion is assumed,

$$
\left.J=\frac{1}{2} \sum_{j=1}^{N}\left(y_{d}(k+j)-\hat{y}(k+j)\right)^{\mathrm{T}} \mathbf{Q}_{p}\left(y_{d}(k+j)\right)-\hat{y}(k+j)\right)+\sum_{j=1}^{N}\left(\Delta u_{m p c}(k+j)\right)^{\mathrm{T}} \mathbf{R}_{p}\left(\Delta u_{m p c}(k+j)\right),
$$

where $y_{d}(k+j), j=1,2, \ldots, N$ is the pressure reference profile and $N$ the prediction horizon, and $\mathbf{Q}_{p}$ and $\mathbf{R}_{p}$ are non-negative definite matrices. Index (16) can be written as

$$
J=\frac{1}{2} \hat{\mathbf{Y}}_{h}^{T}(k) \mathbf{Q}_{p} \hat{\mathbf{Y}}_{h}(k)+\frac{1}{2} \Delta \mathbf{U}_{m p c}^{T}(k) \mathbf{R}_{p} \Delta \mathbf{U}_{m p c}(k),
$$

then the solution minimizing performance index (11) may then be obtained by solving

$$
\frac{\partial J}{\partial \Delta \mathbf{U}_{m p c}}=0 .
$$

A direct off-line computation may be obtained explicitly as

$$
\Delta \mathbf{U}_{m p c}=\left(\mathbf{F}_{1 p}^{\mathrm{T}} \mathbf{Q}_{p} \mathbf{F}_{1 p}+\mathbf{R}_{p}\right)^{-1}\left(\mathbf{F}_{1 p}^{\mathrm{T}} \mathbf{Q}_{p}\left(\mathbf{Y}_{d_{p}}(k)-\mathbf{G}_{p} \mathbf{z}(k)-\mathbf{F}_{2 p} u_{m p c}(k-1)\right)\right),
$$

${ }_{43}$ where $\mathbf{Y}_{d_{p}}(k)$ is the desired output column vector. For further details see [13]. 


\section{4. A stability sufficient constructive condition in GMPC}

45 Theorem 1. Let us take the discrete SISO linear system into consideration:

$$
\begin{aligned}
\mathbf{z}(k+1) & =\mathbf{A}_{k} \mathbf{z}(k)+\mathbf{B}_{k} u_{m p c}(k), \\
y(k) & =\mathbf{H}_{k} \mathbf{z}(k)
\end{aligned}
$$

obtained by a discretisation of a linear continuous system using a sampling time which equals $T_{s} . u_{m p c}(k)$ represents the first element of the vector of the optimal solution as calculated in [13] for the GMPC considering the following cost function:

$$
\begin{aligned}
J=\frac{1}{2} \sum_{j=1}^{N}\left(y_{d}(k+j)-\hat{y}(k+j)\right)^{\mathrm{T}} \mathbf{Q}_{p}\left(y_{d}(k+j)-\hat{y}(k+j)\right)+ & \\
& \sum_{j=1}^{N}\left(\Delta u_{m p c}(k+j-1)\right)^{\mathrm{T}} \cdot \mathbf{R}_{p} \Delta u_{m p c}(k+j-1),
\end{aligned}
$$

where $y_{d}(k+j), j=1,2, \ldots, N$ is the position reference trajectory and $N$ is the prediction horizon, and $\mathbf{Q}_{p}$ and $\mathbf{R}_{p}$ are non-negative definite matrices. Furthermore, the solution minimizing performance index (16) may be obtained by solving:

$$
\frac{\partial J}{\partial \Delta \mathbf{U}_{m p c}}=0 .
$$

It is known for instance from [13] that the optimal solution is:

$$
u_{m p c}(k)=\left(\mathbf{F}_{1 p}^{\mathrm{T}} \mathbf{Q}_{p} \mathbf{F}_{1 p}+\mathbf{R}_{p}\right)^{-1} \mathbf{F}_{1 p}^{\mathrm{T}} \mathbf{Q}_{p}\left(\mathbf{Y}_{d_{p}}(k)-\mathbf{G}_{p} \mathbf{z}(k)-\mathbf{F}_{2 p} u_{m p c}(k-1)\right),
$$

where $\mathbf{Y}_{d_{p}}(k)$ and $\mathbf{Y}_{p}(k)$ are the desired output column vector and the measured or observed output vector. Matrices $\mathbf{Q}_{p}$ and $\mathbf{R}_{p}$ are diagonal and positively defined. Under the technical hypotheses that $\mathbf{Q}_{p}=\mathbf{I}$, and $\mathbf{H}_{k}^{\mathrm{T}} \mathbf{H}=\mathbf{I}$ are under the assumption

i) $r_{(1,1)}>>T_{s}^{2}$, where $r_{(1,1)}$ represents the first diagonal element of matrix $\mathbf{R}_{p}$.

then $\forall r_{(1,1)}$ such that:

$$
r_{(1,1)}>\mathbf{B}_{k} \mathbf{B}_{k}^{\mathrm{T}} \frac{\left\|\mathbf{A}_{k}\right\|_{2}}{1-\left\|\mathbf{A}_{k}\right\|_{2}},
$$

where $\left\|\mathbf{A}_{k}\right\|_{2}$ represents the maximal eigenvalue of matrix $\sqrt{\mathbf{A}^{T} \mathbf{A}}$ and

$$
\left\|\mathbf{A}_{k}-\mathbf{B}_{k}\left(\left(\mathbf{F}_{1 p}^{\mathrm{T}} \mathbf{Q}_{p} \mathbf{F}_{1 p}+\mathbf{R}_{p}\right)^{-1} \mathbf{F}_{1 p}^{\mathrm{T}} \mathbf{Q}_{p} \mathbf{G}_{p}\right)\right\|_{2}<\left\|\mathbf{A}_{k}+\mathbf{B}_{k}\left(\left(\mathbf{F}_{1 p}^{\mathrm{T}} \mathbf{Q}_{p} \mathbf{F}_{1 p}+\mathbf{R}_{p}\right)^{-1} \mathbf{F}_{1 p}^{\mathrm{T}} \mathbf{Q}_{p} \mathbf{G}_{p}\right)\right\|_{2},
$$

then the system (14) is asymptotically stable.

Proof 1. For sake of brevity just one prediction step is considered, then:

$$
\begin{aligned}
& \mathbf{F}_{1 p}=\left[\mathbf{H}_{k} \mathbf{B}_{k}\right], \\
& \mathbf{F}_{2 p}=\left[\mathbf{H}_{k} \mathbf{B}_{k}\right], \\
& \mathbf{G}_{p}=\left[\mathbf{H}_{k} \mathbf{A}_{k}\right] .
\end{aligned}
$$

Combining Eq. (14) with (18), this expression is got:

$$
\mathbf{z}(k+1)=\mathbf{A}_{k} \mathbf{z}(k)+\mathbf{B}_{k}\left(\left(\mathbf{F}_{1 p}^{\mathrm{T}} \mathbf{Q}_{p} \mathbf{F}_{1 p}+\mathbf{R}_{p}\right)^{-1} \mathbf{F}_{1 p}^{\mathrm{T}} \mathbf{Q}_{p}\left(\mathbf{Y}_{d_{p}}(k)-\mathbf{G}_{p} \mathbf{z}(k)-\mathbf{F}_{2 p} u_{m p c}(k-1)\right)\right),
$$


6 of 10

which is equivalent to write:

$$
\begin{aligned}
\mathbf{z}(k+1)=\left(\mathbf{A}_{k}-\mathbf{B}_{k}\left(\left(\mathbf{F}_{1 p}^{\mathrm{T}} \mathbf{Q}_{p} \mathbf{F}_{1 p}+\mathbf{R}_{p}\right)^{-1} \mathbf{F}_{1 p}^{\mathrm{T}} \mathbf{Q}_{p} \mathbf{G}_{p}\right)\right) \mathbf{z}(k)+ \\
\mathbf{B}_{k}\left(\left(\mathbf{F}_{1 p}^{\mathrm{T}} \mathbf{Q}_{p} \mathbf{F}_{1 p}+\mathbf{R}_{p}\right)^{-1} \mathbf{F}_{1 p}^{\mathrm{T}} \mathbf{Q}_{p}\left(\mathbf{Y}_{d_{p}}(k)\right)\right) \\
-\mathbf{B}_{k}\left(\left(\mathbf{F}_{1 p}^{\mathrm{T}} \mathbf{Q}_{p} \mathbf{F}_{1 p}+\mathbf{R}_{p}\right)^{-1} \mathbf{F}_{1 p}^{\mathrm{T}} \mathbf{Q}_{p}\left(\mathbf{F}_{2 p}(k)\right)\right) .
\end{aligned}
$$

If

$$
r_{(1,1)}>\mathbf{B}_{k} \mathbf{B}_{k}^{\mathrm{T}} \frac{\left\|\mathbf{A}_{k}\right\|_{2}}{1-\left\|\mathbf{A}_{k}\right\|_{2}}
$$

${ }_{52}$ considering that scalar $r_{(1,1)}>0$ and scalar $\mathbf{B}_{k} \mathbf{B}_{k}^{\mathrm{T}}>0$, then:

$$
0<\left\|\mathbf{A}_{k}\right\|_{2}+r_{(1,1)}^{-1} \mathbf{B}_{k} \mathbf{B}_{k}^{\mathrm{T}}\left\|\mathbf{A}_{k}\right\|_{2}<1
$$

s3 Recalling that $\mathbf{H}_{k}^{\mathrm{T}} \mathbf{H}=\mathbf{I}$

$$
0<\left\|\mathbf{A}_{k}\right\|_{2}+r_{(1,1)}^{-1} \mathbf{B}_{k} \mathbf{B}_{k}^{\mathrm{T}} \mathbf{H}_{k}^{\mathrm{T}} \mathbf{H}\left\|\mathbf{A}_{k}\right\|_{2}<1
$$

54 and thus

$$
0<\left\|\mathbf{A}_{k}\right\|_{2}+r_{(1,1)}^{-1}\left\|\mathbf{B}_{k} \mathbf{B}_{k}^{\mathrm{T}} \mathbf{H}_{k}^{\mathrm{T}} \mathbf{H} \mathbf{A}_{k}\right\|_{2}<1 .
$$

${ }_{55}$ Being matrix $\mathbf{F}_{1 p}$ defined as in (21) and $\mathbf{G}_{1 p}$ defined in (23), it is known that matrix $\mathbf{B}_{k}$ is proportional $T_{s}$

56 then considering that $\mathbf{R}_{p}=r_{(1,1)}$, choosing a suitable $r_{(1,1)}>>T_{s}$ and considering that $\mathbf{Q}_{p}=\mathbf{I}$ (technical

57 hypothesis), the following condition can be derived:

$$
0<\left\|\mathbf{A}_{k}\right\|_{2}+\|\left(\mathbf{B}_{k}\left(\left(\mathbf{F}_{1 p}^{\mathrm{T}} \mathbf{Q}_{p} \mathbf{F}_{1 p}+\mathbf{R}_{p}\right)^{-1} \mathbf{F}_{1 p}^{\mathrm{T}} \mathbf{Q}_{p} \mathbf{G}_{p}\right) \|_{2}<1\right.
$$

$$
\begin{aligned}
0<\left\|\mathbf{A}_{k}-\mathbf{B}_{k}\left(\left(\mathbf{F}_{1 p}^{\mathrm{T}} \mathbf{Q}_{p} \mathbf{F}_{1 p}+\mathbf{R}_{p}\right)^{-1} \mathbf{F}_{1 p}^{\mathrm{T}} \mathbf{Q}_{p} \mathbf{G}_{p}\right)\right\|_{2} \\
<\left\|\mathbf{A}_{k}+\mathbf{B}_{k}\left(\left(\mathbf{F}_{1 p}^{\mathrm{T}} \mathbf{Q}_{p} \mathbf{F}_{1 p}+\mathbf{R}_{p}\right)^{-1} \mathbf{F}_{1 p}^{\mathrm{T}} \mathbf{Q}_{p} \mathbf{G}_{p}\right)\right\|_{2} \\
\quad<\left\|\mathbf{A}_{k}\right\|_{2}+\left\|\mathbf{B}_{k}\left(\left(\mathbf{F}_{1 p}^{\mathrm{T}} \mathbf{Q}_{p} \mathbf{F}_{1 p}+\mathbf{R}_{p}\right)^{-1} \mathbf{F}_{1 p}^{\mathrm{T}} \mathbf{Q}_{p} \mathbf{G}_{p}\right)\right\|_{2}<1 .
\end{aligned}
$$

$59 \quad$ To conclude

$$
0<\left\|\mathbf{A}_{k}\right\|-\left\|\mathbf{B}_{k}\left(\left(\mathbf{F}_{1 p}^{\mathrm{T}} \mathbf{Q}_{p} \mathbf{F}_{1 p}+\mathbf{R}_{p}\right)^{-1} \mathbf{F}_{1 p}^{\mathrm{T}} \mathbf{Q}_{p} \mathbf{G}_{p}\right)\right\|_{2}<1 .
$$

68

The constraint in (26) states a plausible condition on the controller. Stability represents the necessary condition of the optimality of a controlled system. In order to interpret the result let us observe a mechanical system including a mass-spring system in which it is known that the eigenvalue can variate in the whole real and complex domain as a function of the mass which states the system inertia. If mass $m \rightarrow \infty$, then, because of the discretisation and according to the Landau notation, $\left(\| \lambda_{\max }\left(\mathbf{A}_{k}\right)-1 \mid\right) \rightarrow 0$ with $\mathcal{O}\left(\left|\lambda_{\max }\left(\mathbf{A}_{k}\right)-1\right|\right)=\mathcal{O}\left(\frac{1}{m}\right)$. In the meantime $\mathcal{O}\left(\mathbf{B}_{k} \mathbf{B}_{k}^{\mathrm{T}}\right)=\mathcal{O}\left(\frac{1}{m^{2}}\right)$. For a very slow system, according to (26), $r_{(1,1)} \rightarrow 0$, parameter $r_{(1,1)}$ is present in the denominator function of the optimal solution in (18) and in this case, small values of $r_{(1,1)}$ are devoted to speed up the system. If mass $m \rightarrow 0$, then, because of the discretisation, $\mathcal{O}\left(\left|\lambda_{\max }\left(\mathbf{A}_{k}\right)\right|\right)=\mathcal{O}\left(\frac{1}{m}\right)$, but in the meantime $\mathbf{B}_{k} \mathbf{B}_{k}^{\mathrm{T}} \rightarrow \infty$ with $\mathcal{O}\left(\mathbf{B}_{k} \mathbf{B}_{k}^{\mathrm{T}}\right)=\mathcal{O}\left(\frac{1}{m^{2}}\right)$. For a very fast system $r_{(1,1)} \rightarrow \infty$, parameter $r_{(1,1)}$ is 
and

$$
\left|u_{m p c}(k)\right| \leq U_{\max } \forall k,
$$

then (33) with the input saturation defined in (35) being asymptotically stable and its input avoiding the constraint if condition (26) holds together with the input limitation. The following condition summarizes the result:

$$
r_{(1,1)}>\max \left\{\frac{\mathbf{B}_{k} \mathbf{B}_{k}^{\mathrm{T}}\left\|\mathbf{Y}_{d_{p}}(k)\right\|_{2}}{U_{\max }}+\frac{\left\|\mathbf{B}_{k} \mathbf{F}_{1 p} \mathbf{Q}_{p} \mathbf{F}_{2 p}\right\|_{2}}{U_{\max }}\left|u_{m p c}(k-2)\right|, \quad \mathbf{B}_{k} \mathbf{B}_{k}^{\mathrm{T}} \frac{\left\|\mathbf{A}_{k}\right\|_{2}}{1-\left\|\mathbf{A}_{k}\right\|_{2}}\right\} .
$$

Proof 2. The demonstration is straightforward just considering that:

$$
\left|u_{m p c}(k)\right|<\left|u_{m p c}(k)\right|<U_{m a x} \forall k
$$

and thus it is enough that the following condition holds:

$$
\left\|\mathbf{B}_{k}\left(\left(\mathbf{F}_{1 p}^{\mathrm{T}} \mathbf{Q}_{p} \mathbf{F}_{1 p}+\mathbf{R}_{p}\right)^{-1} \mathbf{F}_{1 p}^{\mathrm{T}} \mathbf{Q}_{p}\left(\mathbf{Y}_{d_{p}}(k)\right)\right)-\mathbf{B}_{k}\left(\mathbf{F}_{1 p}^{\mathrm{T}} \mathbf{Q}_{p} \mathbf{F}_{1 p}+\mathbf{R}_{p}\right)^{-1} \mathbf{F}_{1 p} \mathbf{Q}_{p} \mathbf{F}_{2 p} u_{m p c}(k-1)\right\|_{2} \leq U_{\max } .
$$

In fact, using similar considerations as before, the following expression is obtained:

$$
\left\|\mathbf{B}_{k} r_{11}^{-1} \mathbf{F}_{1 p}^{\mathrm{T}} \mathbf{Q}_{p}\left(\mathbf{Y}_{d_{p}}(k)\right)\right\|_{2}+\left|\mathbf{B}_{k} \mathbf{F}_{1 p} \mathbf{Q}_{p} \mathbf{F}_{2 p}\right| \leq U_{\max }
$$

and thus, including also the stability condition, condition (36) follows here below again:

$$
r_{(1,1)}>\max \left\{\frac{\mathbf{B}_{k} \mathbf{B}_{k}^{\mathrm{T}}\left\|\mathbf{Y}_{d_{p}}(k)\right\|_{2}}{U_{\max }}+\frac{\left\|\mathbf{B}_{k} \mathbf{F}_{1 p} \mathbf{Q}_{p} \mathbf{F}_{2 p}\right\|_{2}}{U_{\max }}\left|u_{m p c}(k-1)\right|, \quad \mathbf{B}_{k} \mathbf{B}_{k}^{\mathrm{T}} \frac{\left\|\mathbf{A}_{k}\right\|_{2}}{1-\left\|\mathbf{A}_{k}\right\|_{2}}\right\} .
$$

It is possible to observe that for large values of $U_{\max }$ the condition on the input barrier in the cost function (16) with weight $r_{(1,1)}$ is not so restrictive, so larger inputs are allowed. For small values of $U_{\max }$ the input barrier limits the values of the input and no large input values are allowed.

\section{Simulation results}

Concerning the simulation results, it is to be clarified that function $m_{i}(t)$ is a stepwise constant function with $m_{i}(t)=0.086\left(\mathrm{~kg} / \mathrm{sec}\right.$.) or $m_{i}(t)=0$ and in the simulated case $m_{i}(t)=0.086(\mathrm{~kg} / \mathrm{sec}$. $)$ is considered. As already explained, in the simulations two cases should be distinguished: weak anti-saturating action:

$$
r_{(1,1)}>\frac{\mathbf{B}_{k} \mathbf{B}_{k}^{\mathrm{T}}\left\|\mathbf{Y}_{d_{p}}(k)\right\|_{2}}{U_{\max }} .
$$


and strong anti-saturating action:

$$
r_{(1,1)}>>\frac{\mathbf{B}_{k} \mathbf{B}_{k}^{\mathrm{T}}\left\|\mathbf{Y}_{d_{p}}(k)\right\|_{2}}{U_{\max }}
$$

Fig. 2 shows the controlled pressure which represents the main result. In case of anti-windup action being relatively weak, the anti-windup controller needs time to reestablish the control loop. As already explained, during the windup effect the feedback control is broken so the absence of the feedback control action can clarify the long permanence of the pressure at negative values. In particular, from Fig. 3 which represents the mass flow $\frac{d m(t)}{d t}=m_{i n}(t)-m_{0}(t)(\mathrm{kg} / \mathrm{sec})$ it is visible how this function is consequent with the relation:

$$
\frac{d m(t)}{d t} \approx \frac{d p(t)}{d t}
$$

Moreover, considering the following equation again:

$$
\frac{d m(t)}{d t}=m_{\text {in }}(t)-m_{0}(t)
$$

to activate the process a strong initial action through the mass flow $m_{0}(t)$ is required. Observing these two figures it is possible too see that in the case of strong anti saturating action represented in Fig. 5 the controlled system through the anti-windup scheme comes out from the saturation state very quickly with a resulting faster dynamics. The resulting faster dynamics are obtained thanks to the stronger anti-windup action which allows to reactivate the control loop very quickly. As already explained, when the saturation occurs the control loop is open and no feedback control is present.

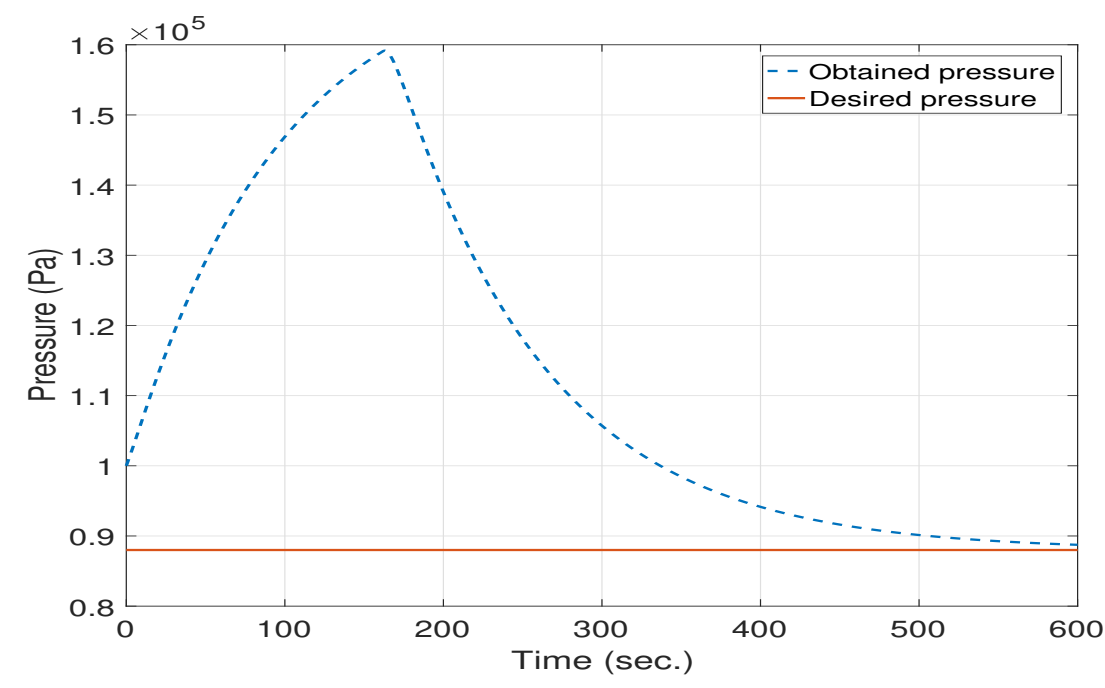

Figure 2. Desired and obtained pressure with weak anti-saturating action

\section{Conclusion}

One of the most important problems in the context of optimisation using LMPC is represented by conservative conditions on the stability. This paper presents a sufficient and constructive condition for the stability of an LGMPC calculating lower bounds of the element of matrix $\mathbf{R}$ which represent the weights of the input in a typical given cost function. A physical interpretation of the result is given on the light of some physical considerations. An illustrative example is provided in which a recover water process is taken into consideration to test the proposed results through computer simulations. 


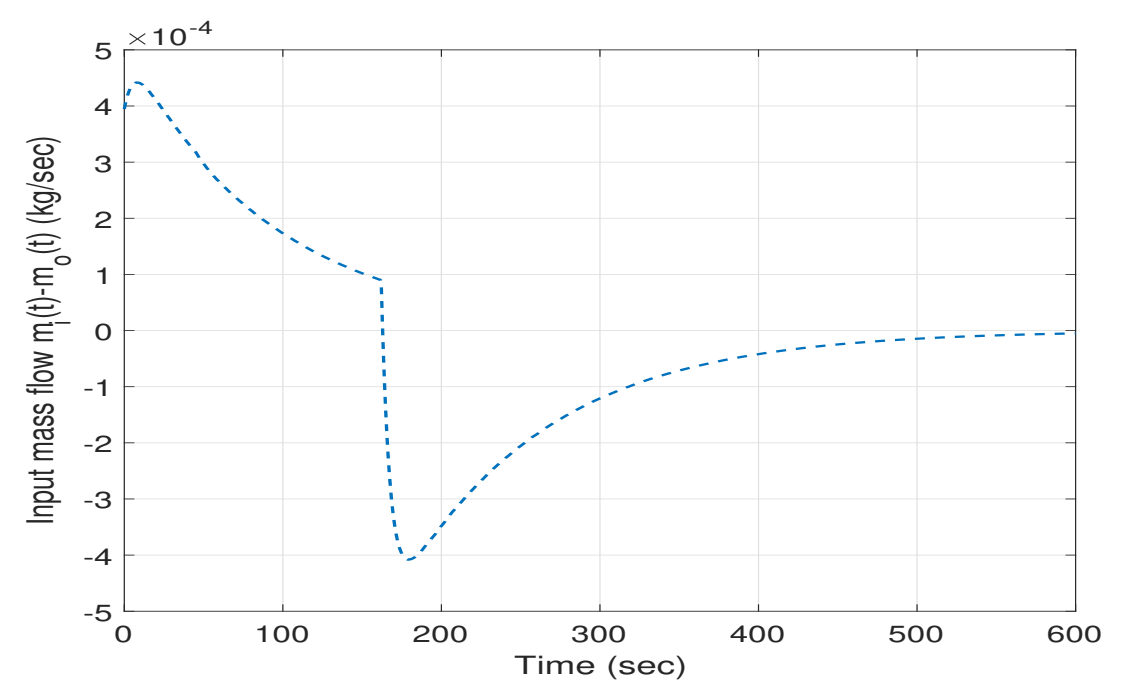

Figure 3. Mass flow $\frac{m(t)}{d t}=m_{\text {in }}(t)-m_{0}(t)(\mathrm{kg} / \mathrm{sec})$ with weak anti-saturating action

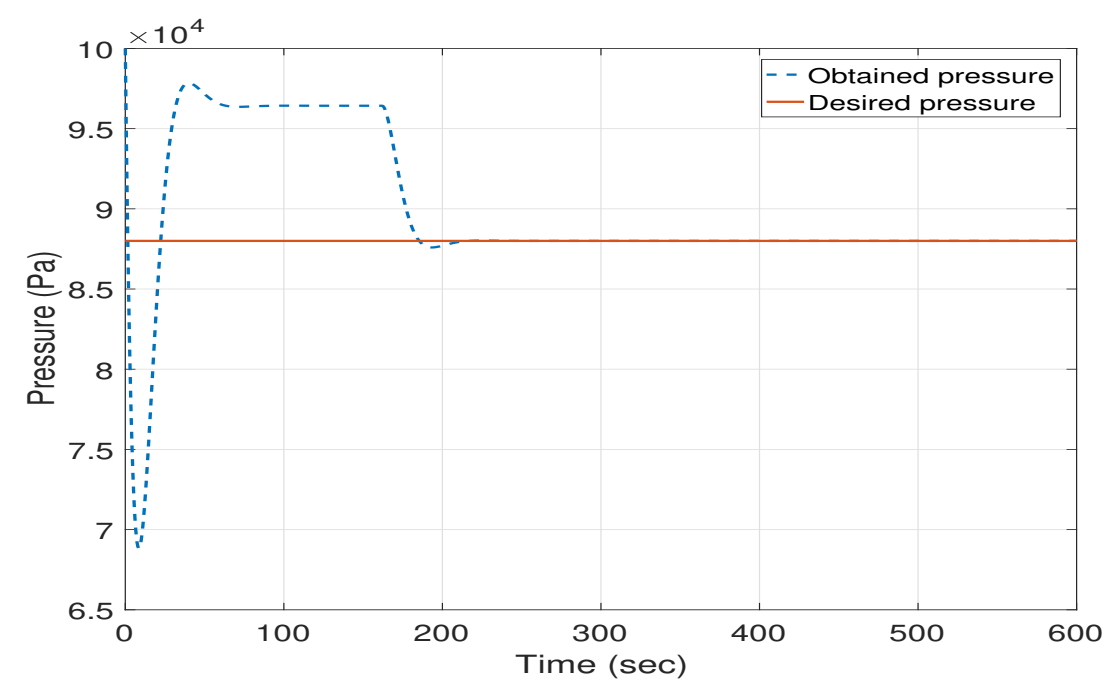

Figure 4. Desired and obtained pressure with strong anti-saturating action

\section{References}

1. Mercorelli, P. A switching Kalman Filter for sensorless control of a hybrid hydraulic piezo actuator using MPC for camless internal combustion engines. Proceedings of the IEEE International Conference on Control Applications, 2012, pp. 980-985.

2. Mercorelli, P. A switching model predictive control for overcoming a hysteresis effect in a hybrid actuator for camless internal combustion engines. Proceedings of the IEEE PRECEDE 2011 - International Workshop on Predictive Control of Electrical Drives and Power Electronics, 2011, pp. 10-16.

3. Qin, S.; Badgwell, T. A survey of industrial model predictive control technology. Control Engineering Practice 2003, 11, 733-764.

4. Bolognani, S.; Peretti, L.; Zigliotto, M. Design and implementation of model predictive control for electrical motor drives. IEEE Transactions on Industrial Electronics 2009, 56, 1925-1936.

5. Neelakantan, V.; Washington, G.; Bucknor, N. Model Predictive Control of a Two Stage Actuation System using Piezoelectric Actuators for Controllable Industrial and Automotive Brakes and Clutches. J. Intel. Mater. Syst. and Struct 2008, 19, 845-857. 


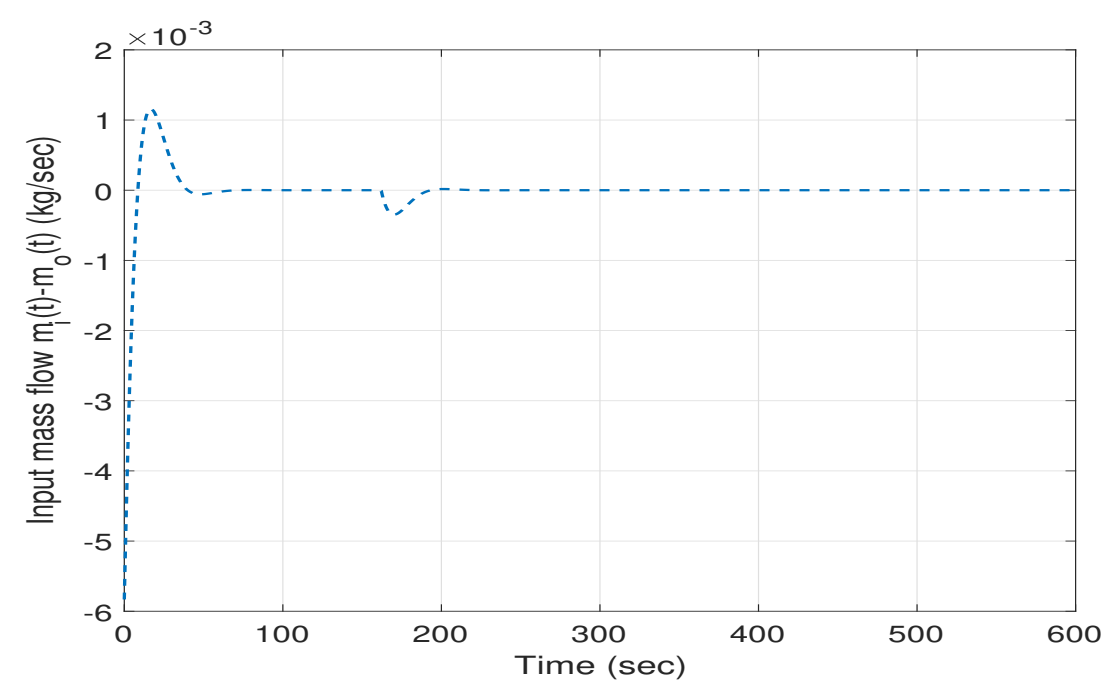

Figure 5. Mass flow $\frac{d m(t)}{d t}=m_{\text {in }}(t)-m_{0}(t)(\mathrm{kg} / \mathrm{sec})$ with strong anti-saturating action

6. Hu, Z.; Farson, D. Design of a waveform tracking system for a piezoelectric actuator. Proc. Inst. Mech. Eng., J. Syst. Control Eng., 2008, Vol. 222, pp. 11-21.

7. Bemporad, A.; Morari, M.; Dua, V.; Pistikopoulos, E.N. The explicit linear quadratic regulator for constrained systems. Automatica 2002, 38, 3-20.

8. Pedret, C.; Poncet, A.; Stadler, K.; Toller, A.; Glattfelder, A.; Bemporad, A.; Morari, M. Model-varying predictive control of a nonlinear system. Internal report in Computer Science Dept. ETSE de la Universitat Autònoma de Barcelona 2000.

9. Liu, Y.C.; Lin, C.Y. Model Predictive Control with Integral Control and Constraint Handling for Mechatronic Systems. Proc. of the 2010 International Conference on Modelling, Identification and Control; , 2010; pp. 424-429.

10. Mercorelli, P.; Goes, J.; Halbe, R. A Lyapunov based PI controller with an anti-windup scheme for a purification process of potable water. 2014 International Conference on Control, Decision and Information Technologies (CoDIT), 2014, pp. 578-583.

11. Mercorelli, P. An Optimal and Stabilising PI Controller with an Anti-windup Scheme for a Purification Process of Potable Water. 16th IFAC Workshop on Control Applications of Optimization CAO'2015, 2015, pp. 259-264.

12. Mercorelli, P. A sufficient asymptotic stability condition in generalised model predictive control to avoid input saturation. Lecture Notes in Electrical Engineering 2019, 489, 251-257.

13. Sunan, H.; Kiong, T.; Heng, L. Applied Predictive Control; Springer-Verlag London: Printed in Great Britain, 2002. 